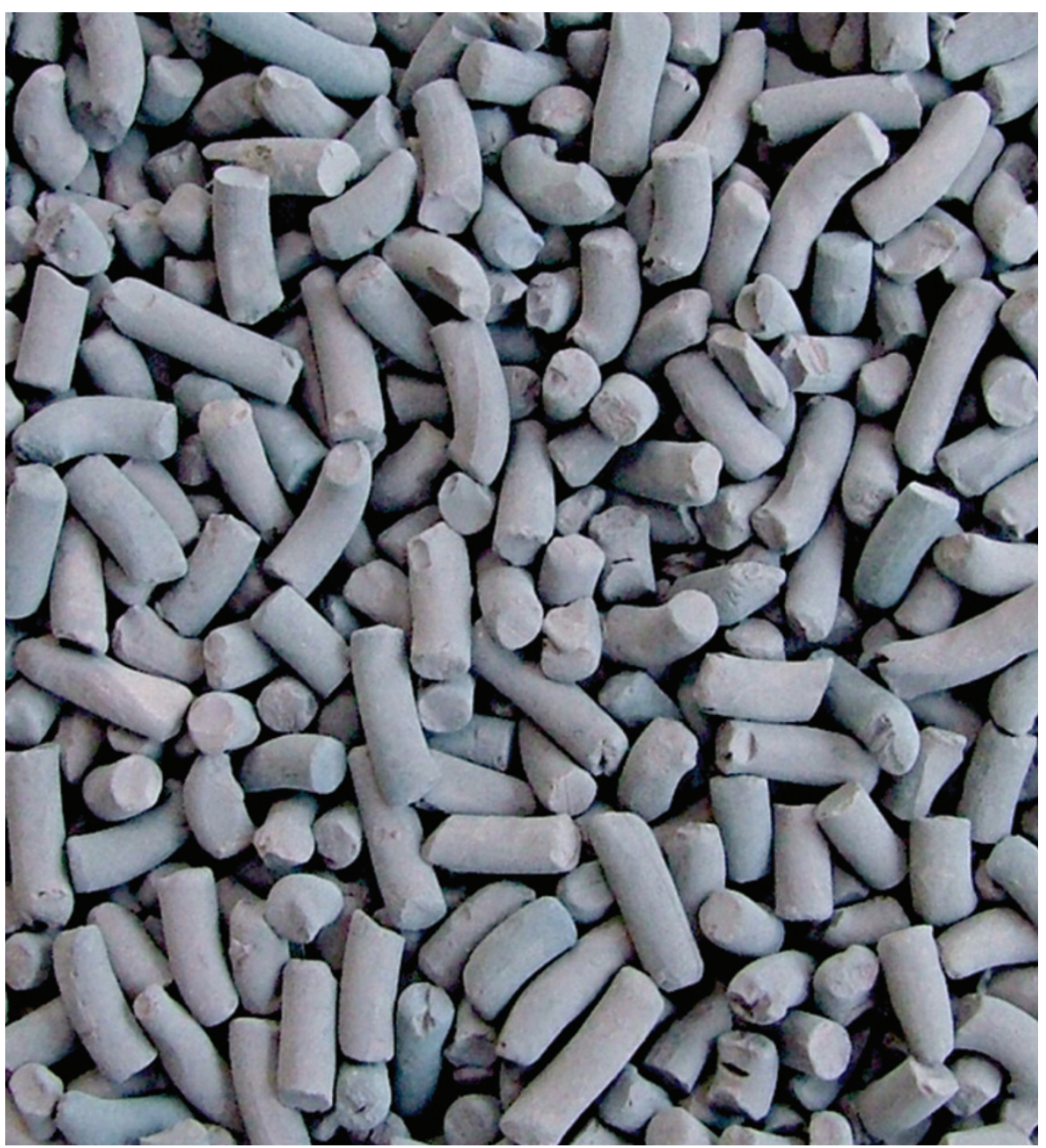

A material combining gold and titanium dioxide can scrub pollutants from smoke-filled environments.

\title{
CATALYSIS
}

\section{The accelerator}

\section{Gold can speed up a multitude of chemical reactions - so why isn't it widely used in industry?}

\section{BY MARK PEPLOW}

C $\mathbf{T}$ adies and gentlemen, start your engines!" The air fills with the poison-

ous fumes pouring from the roaring stock cars lined up on the grid at the Daytona 500 , the most prestigious race of this year's NASCAR Sprint Cup Series. Inside two of the cars, though, the drivers are breathing easy their air supply has been scrubbed clean by a gold catalyst called NanAuCat, which uses ambient oxygen to convert carbon monoxide to carbon dioxide.

This is a little-known application of a catalyst that has taken more than two decades to complete the journey from the lab to the racetrack. Back in 1987, chemist Masatake Haruta of the Government Industrial Research Institute of Osaka, Japan, and colleagues showed that tiny particles of gold were remarkably active, helping to unite carbon monoxide and oxygen to make carbon dioxide.

The idea that gold could hasten chemical reactions was deeply counterintuitive for most chemists. After all, gold is famously inert in its bulk form. It has been used for millennia to make jewellery precisely because it does not tarnish by reacting with oxygen or water.

For inorganic chemist Graham Hutchings, however, Haruta's findings chimed with a discovery of his own. A few years earlier, he was hunting for a catalyst to convert acetylene to vinyl chloride - the starting point for a plethora of plastic products that use polyvinyl chloride. The conventional synthesis relied on a mercury (II) chloride catalyst, a source of environmental mercury pollution. Hutchings, now at Cardiff University, UK, checked the standard electrode potentials of a host of alternative metals - a measurement that describes the metal ion's ability to grab an electron - and realized that gold was almost perfectly suited to cosy up to acetylene and accelerate its reactions.

The discoveries by Hutchings and Haruta set the stage for a boom in research on gold's catalytic properties. "The field is still in exponential growth," says Stephen Hashmi, a gold chemist at the University of Heidelberg, Germany. "Even now, every day, people are finding new reactions that use gold catalysts."

Yet despite the slew of research, these two breakthrough reactions remain gold's greatest hits. And neither has been scaled up for use in large-scale chemical production plants, although Haruta's carbon monoxide oxidation has found a few niche applications. The main stumbling block is economic: although gold is not as costly as some other metal catalysts such as platinum, and can be more effective, it has mostly proven too expensive to displace existing technologies.

But the intensive research effort is finally pinning down exactly how gold speeds up chemical reactions, enabling scientists to boost its activity and durability enough to make it suitable for industrial work. Along the way, learning how gold behaves at the nanoscale has led to diverse applications in medicine (see 'The new gold standard', page S14), electronics (see 'Trick of the light', page S8) and motoring.

\section{BREATHE EASY}

Away from the thrills of the Daytona 500, gold can be found oxidizing carbon monoxide in a more mundane location: the exhaust pipe of a Fiat.

Platinum is the active ingredient in most catalytic converters, which help to oxidize carbon monoxide, unburned hydrocarbons and other engine emissions in order to reduce pollution. But in 2011, Fiat began installing in some of its diesel vehicles a gold-palladium catalyst NSGold - developed by start-up Nanostellar of Redwood, California.

Nanostellar's catalyst would have seemed a bargain five years ago when the cost of platinum passed US $\$ 70$ per gram, more than twice the price of gold. But by the end of 2012, the global economic crisis had squeezed car production, reducing the need for platinum and driving down its price. At the same time, the price of gold rose rapidly as investors sought a safe haven for their cash. Gold now costs almost the same as platinum, in the $\$ 50-55$ per gram range. "The advantage has pretty much vanished," says materials scientist Kyeongjae Cho of the University of Texas, Dallas, who developed the catalyst and co-founded the company. It is unclear whether Fiat will install any more gold catalytic converters. 
Other companies are still betting on the same reaction, albeit in a different context: fire safety respirators. Project AuTEK, a gold technology effort run by South African mining technology company Mintek, in partnership with other mining-industry players, has developed a range of gold catalysts embedded on metal oxides that can scrub pollutants from smoke-filled environments.

Although chemists are still debating exactly how Haruta's reaction works, it seems that the metal oxides bind and activate oxygen from the air, which can then react with carbon monoxide molecules stuck to neighbouring clusters of gold atoms. These can outperform conventional catalysts used in emergency respirators that help people escape burning buildings. Hooded respirators typically bear a cartridge containing Hopcalite, a catalyst that is very good at speeding up carbon monoxide oxidation - but only when dry, explains Nathan Fredericks, a senior researcher on Project AuTEK. That's because water molecules block the active sites of the Hopcalite catalyst, explains Stan Golunski, who studies gold chemistry at Cardiff University. Smoky buildings tend to be humid so the cartridge needs to contain a protective bed to stop the damp air damaging the catalyst, as well as extra sacrificial Hopcalite. These additions result in a bulky, 200-300-millilitre unit.

Gold catalysts, however, are moisture tolerant in up to $95 \%$ humidity and, says Fredericks, have a much higher activity than Hopcalite, resulting in better-performing cartridges that are one-tenth the size. What's more, gold atoms can quickly cause water to react with carbon monoxide and eject the products, leaving them free for further reactions.

Novax Material and Technology of Luzhu, Taiwan, for example, already uses this sort of gold catalyst technology in a civilian safety respirator. However, the more recent versions of AuTEK's 2-5-nanometre-wide catalyst particles have higher air flow rates, so firefighters should be able to use them without breathing restrictions, says Fredericks.

\section{COST CONCERNS}

As with all potential applications of gold catalysts, however, "the biggest hurdle is price", admits Fredericks. If that hurdle can be cleared - either by price variations or by improving gold's efficiency - the metal could find new roles in industrial chemical production. Advocates point out that there is already one widely known example of gold being used in this way: the production of the polymer precursor vinyl acetate, an application that actually predates Haruta's and Hutchings' discoveries.

For the past decade or so, the preferred catalyst for producing vinyl acetate has been an alloy of $95 \%$ palladium and $5 \%$ gold, says Golunski. But this idea dates back to the late 1960s - a patent on a similar catalytic alloy of gold and palladium to make vinyl acetate was issued in 1973.

\section{CORNER CATALYSIS}

Gold atoms sitting at the corners of catalyst particles are most able to participate in a chemical reaction. So using smaller clusters of gold atoms can maximize the number of these active atoms.

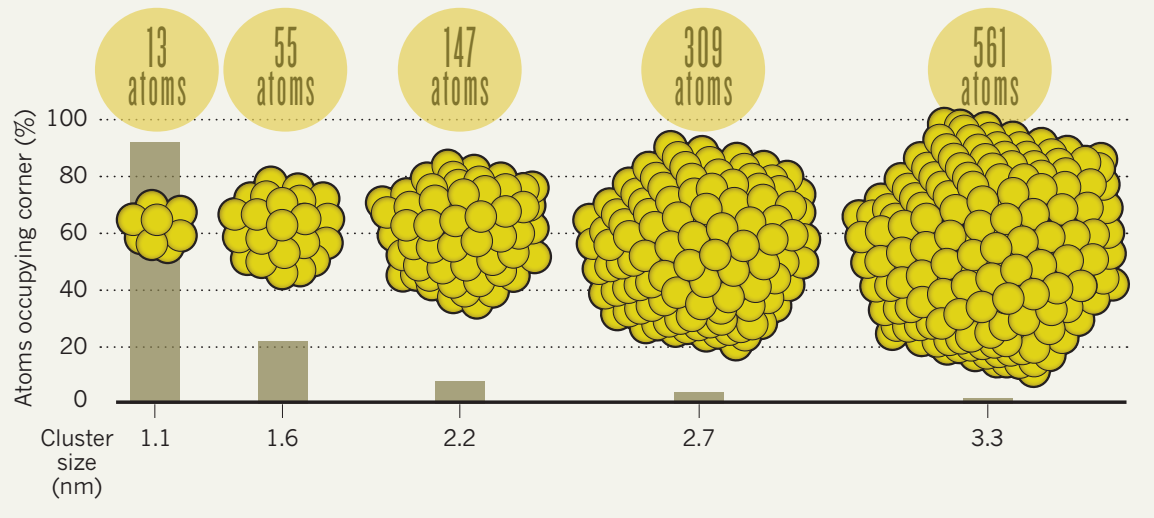

It's a reminder of the sometimes glacial pace of change in the chemicals industry. Many high-volume processes are decades old and built around the use of a specific catalyst, so unless gold can be easily substituted for existing catalysts, it is unlikely that any efficiency savings it offers could offset the enormous costs of building entirely new process infrastructure.

The most promising large-scale applications will be found where gold has little established competition, Golunski says. For example, the stream of hydrogen gas that powers certain types of fuel cell often contains traces of carbon monoxide, which can damage the cells. Chemist Xiao Cheng Zeng of the University of Nebraska-Lincoln has shown that clusters of just 16 to 35 gold atoms can selectively oxidize the carbon monoxide without touching the hydrogen itself, thus extending the fuel cell's lifetime.

\section{SMALL IS BEAUTIFUL}

Gold would become a far more cost-effective prospect for industrial catalysis if chemists could improve its activity and durability. Avelino Corma, a gold chemist at the Polytechnic University of Valencia in Spain, last year gave those efforts a boost by showing that clusters of just three to ten gold atoms could efficiently catalyse a previously known reaction of water with alkynes - molecules containing a carbon-carbon triple bond, such as acetylene.

Each cluster could typically catalyse about 100,000 reactions per hour, among the highest ever reported for a gold catalyst. Moreover, each cluster can complete roughly 10 million reactions before losing its potency - a longevity at least ten times greater than that of any other gold catalyst. This performance makes Corma's catalysts a more realistic prospect for use in industry, says Hashmi. "He's in that league now."

Corma's discovery continues the most notable trend in the field: smaller particle size is better. Haruta's work in the 1980s used gold nanoparticles roughly 2-5 nanometres wide, containing hundreds of atoms - most of which were hidden inside the particle and did not participate in any chemical reactions. Of the atoms on the surface, the most active sit at corners or edges, where their electrons are most exposed to incoming reactants (see 'Corner catalysis').

Corma's tiny clusters of atoms measure just a few tenths of a nanometre across, so every gold atom effectively sits at a corner. This strategy dramatically reduces the amount of gold needed - or allows it to be alloyed on the exterior of cheaper metal nanoparticles potentially slashing the cost.

Corma's next step is to stabilize the catalyst clusters to keep them active for even longer, potentially giving them a greater advantage over existing catalysts. In unpublished work, he says, he has stuck his gold clusters to a solid, inert material that makes the catalyst more durable - and more easily recovered after the reaction - while maintaining the same turnover frequency.

If economics or efficiency cannot propel gold catalysts into widespread use, tighter safety regulations might. In 2011, the US National Fire Protection Association issued revised standards for safety respirators worn while fighting wildfires, increasing the duration of protection against carbon monoxide from 30 minutes to 8 hours. Fredericks says protection for this length of time cannot be achieved using current Hopcalite technology, leaving gold catalysis as the only viable option.

Indeed, it was the importance of safety that put gold in pole position at the NASCAR Sprint Cup. Teams have paid much more attention to air purification since former NASCAR winner Rick Mast retired in 2002, citing ill health from chronic carbon monoxide poisoning. In the end, predicts Fredericks, "people will start to use gold catalysts because they'll have to."

Mark Peplow, formerly Nature's news editor, is a freelance science journalist based in Cambridge, UK. 